\title{
Forces and torques on the nanoscale: from measurement to applications
}

Giovanni Volpe 


\title{
Forces and Torques on the Nanoscale: From Measurement to Applications
}

\author{
Giovanni Volpe* \\ Department of Physics, Bilkent University, Cankaya, Ankara 06800, Turkey
}

\begin{abstract}
The possibility of measuring microscopic forces down to the femtonewton range has opened new possibilities in fields such as biophysics and nanophotonics. I will review some of the techniques most often employed, namely the photonic force microscope (PFM) and the total internal reflection microscope (TIRM), which are able to measure tiny forces acting on optically trapped particles. I will then discuss several applications of such nanoscopic forces: from plasmonic optical manipulation, to self-propelled microswimmers, to self-organization in large ensembles of particles.
\end{abstract}

Keywords: Optical tweezers, force, torque, Brownian motion, photonic force microscope, total internal reflection microscopy, plasmonics, nanoscience

\section{INTRODUCTION}

Forces and torques $-\mathrm{a}$ torque is a vector associated with a twisting force that tends to rotate an object - are used to describe physical interactions.

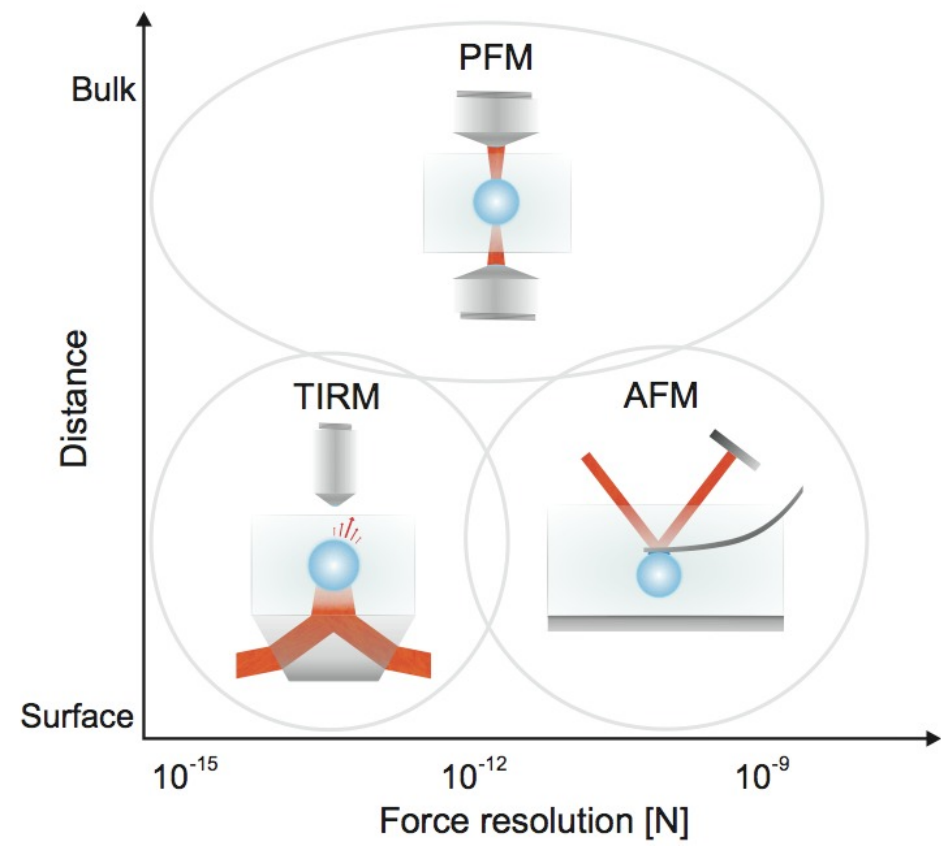

Figure 1. Main techniques to measure forces at the nanoscopic scale. The various force-measurement techniques are classified according to their force resolution and the working conditions - surface/bulk - for which they are best suited: atomic force microscopy (AFM), photonic force microscopy (PFM), and total internal reflection microscopy (TIRM).

* giovanni.volpe@fen.bilkent.edu.tr; http://softmatter.bilkent.edu.tr

Optical Trapping and Optical Micromanipulation IX, edited by Kishan Dholakia, Gabriel C. Spalding, Proc. of SPIE Vol. 8458, 84580F · (C) 2012 SPIE · CCC code: 0277-786/12/\$18 · doi: 10.1117/12.929409 
We all have an intuition about how to deal with our-daily-life forces and torques. In particular, we know how to measure them and how to take advantage of them for our purposes. However, forces and torques are also present at the micrometric and molecular scale. In these cases the behavior of such forces and torques is different from what we are used to: things get much more complex when we move into these weird microscopic domains with less connection with our daily experience. In particular, one thing has to be kept in mind: the thermal noise, responsible for the Brownian diffusion of particles, is omnipresent ${ }^{1}$. Furthermore, when liquid environment are considered, inertial effects are absent at the microscopic scale due to the overwhelming role of viscosity ${ }^{2,3}$. Anyway, we do want to deal with these molecularscale forces and torques, and in particular to measure them. To measure them is crucial for our understanding of the biophysical processes that involve these molecules and for the advancement of nanotechnology.

In this article, I will review the basic techniques to measure microscopic forces, in particular the photonic force microscope (PFM) and the total internal reflection microscope (TIRM). Then, I will present some applications to the fields of biophysics, plasmonics, statistical physics and active systems.

\section{PHOTONIC FORCE MICROSCOPY}

Various techniques have been developed to probe the mechanical properties of microsystems. In the early 90s various kinds of scanning probe microscopy were already established. In 1982 Binnig and coworkers invented the Scanning Tunneling Microscope (STM) ${ }^{4}$, which permitted one to resolve at the atomic level crystallographic structures and organic molecules. The Atomic Force Microscope (AFM) was invented in $1986^{5}$. These instruments have been successfully employed to study biological and nano-fabricated structures, overcoming the traditional diffraction limit of optical microscopes. Furthermore, they developed from pure imaging tools into more general manipulation and measuring tools on the level of single atoms or molecules. However, all these techniques required a macroscopic mechanical device to guide the probe.

The advent in 1986 of the first 3D optical trap paved the way towards new kinds of probes. Ashkin demonstrated that the radiation pressure from a focused laser beam is able to trap small particles ${ }^{6}$. For example, an optical trap is formed when a micron-sized transparent particle whose index of refraction is greater than the surrounding medium is located within a highly focused laser beam. The refracted rays differ in intensity over the volume of the sphere and exert a piconewton scale force on the particle, drawing it towards the region of highest light intensity, and producing an elastic restoring force. The particle settles down near the equilibrium position, but never completely; instead it keeps on jiggling because of the Brownian motion. 


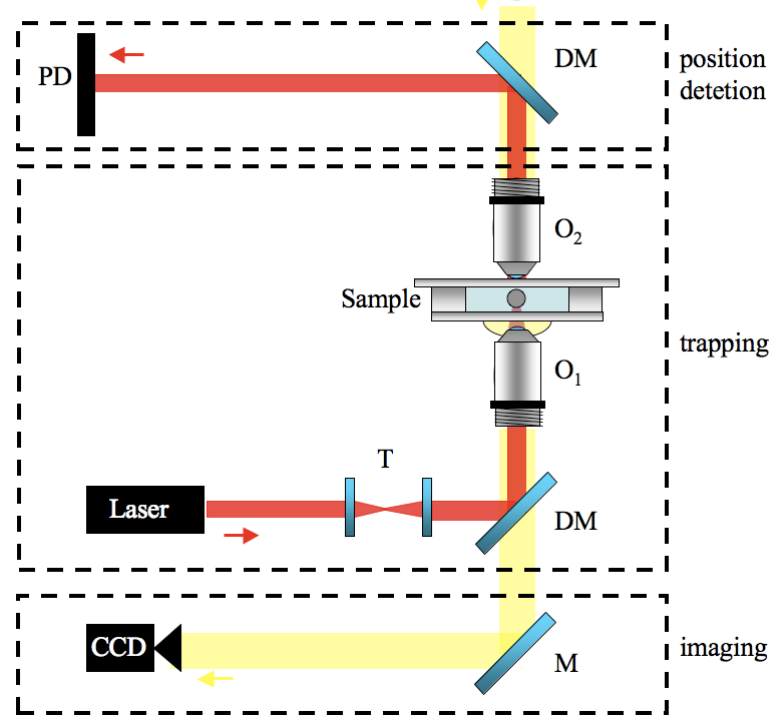

Figure 2. Basic PFM setup. Main components: laser, high-NA objective (O1 ), condenser (O2 ), sample, photodetector (PD), and CCD camera. Other optical components: telescope (T), dichroic mirrors (DM), mirror (M), and illumination light source (LS).

In 1993 Ghislain and coworkers devised a new kind of scanning force microscopy using such an optically trapped microsphere as a probe ${ }^{7}$. This technique was later called Photonic Force Microscope (PFM). The PFM provides the capability of measuring forces in the range from femto- to piconewton. This value is well below the one that can be reached with technique that based on microfabricated mechanical cantilevers ${ }^{8}$. A photonic force microscope (PFM) can measure forces in the range from few femtonewton to several hundred piconewton and it is particularly suited to address forces acting on particles in the bulk. Therefore, it compliments the range of interactions that can be probed by atomic force microscopy (AFM) and total internal reflection microscopy (TIRM).

Photonic force microscopy (PFM) is a technique that relies on a single optical tweezers to measure small forces (piconewtons down to femtonwtons). A typical PFM comprises an optical trap that holds a probe - a dielectric or metallic particle of micrometer size, which randomly moves due to Brownian motion in the potential well formed by the optical trap - and a position sensing system. The analysis of this thermal motion provides information about the local forces acting on the particle 9 . The three-dimensional probe position can be recorded through different devices, which detect the forward or backward scattered light from the particle ${ }^{10}$. The most commonly used are a quadrant photodiode, a position sensing detector, or a camera. PFM permits one to measure forces down to few femtonewton and also torques down to few femtonewton-nanometers ${ }^{11-21}$ at a sampling frequency up to $250 \mathrm{kHz}$ (recently, employing more sophisticated detectors also sampling frequencies up to $75 \mathrm{MHz}$ have been achieved $^{22}$ ).

\section{TOTAL INTERNAL REFLECTION MICROSCOPY}

Total internal reflection microscopy (TIRM) can measure the interaction potentials between a colloidal particle and a wall with femtonewton resolution ${ }^{23,24}$. The equilibrium distribution of the particle-wall separation distance $\mathrm{z}$ is sampled monitoring the intensity I scattered by the Brownian particle under evanescent illumination. 

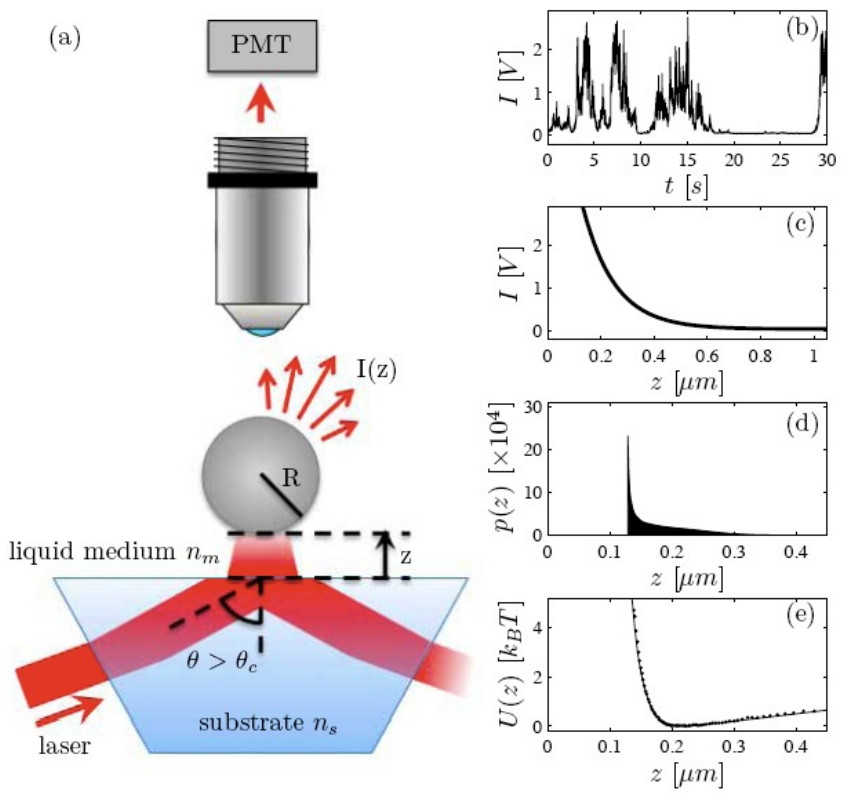

Figure 3. Total Internal Reflection Microscopy (TIRM). (a) Schematic of a typical TIRM setup: a Brownian particle moves in the evanescent electromagnetic field generated by total internal reflection of a laser beam; its scattering is collected by an objective lens; and the scattering intensity is recorded using a photomultiplier (PMT). (b) Typical experimental scattering intensity time-series (polystyrene particle in water, $\mathrm{R}=1.45 \mu \mathrm{m})$. (c) Exponential intensity-distance relation $(\beta=120 \mathrm{~nm})$. $(\mathrm{d})$ Particle position distribution (acquisition time 1200s, sampling rate $500 \mathrm{~Hz}$ ). (e) Experimental (dots) and theoretical (line) potential obtained from the position distribution using the Boltzmann factor. Reproduced from Ref. 25.

From the potential one can determine the distance-resolved interaction potential and corresponding forces with femtonewton resolution. The central point of the data analysis is the a priori knowledge of the relation between the measured scattering intensity I and the corresponding particle distance z. For short penetration depths of the evanescent field, it has been demonstrated that $\mathrm{I}(\mathrm{z}) \exp (-\mathrm{z} / \mathrm{d})$. This, however, poses considerable constraints to the experimental conditions and the range of forces where TIRM can be applied. We introduced a method to experimentally determine $\mathrm{I}(\mathrm{z})$ by making solely use of the distance-dependent hydrodynamic interactions between the particle and the wall ${ }^{25}$. We demonstrated that our method largely extends the range of conditions accessible with TIRM, and even allows measurements on highly reflecting gold surfaces where multiple reflections lead to large deviations from an exponential I(z) relationship.

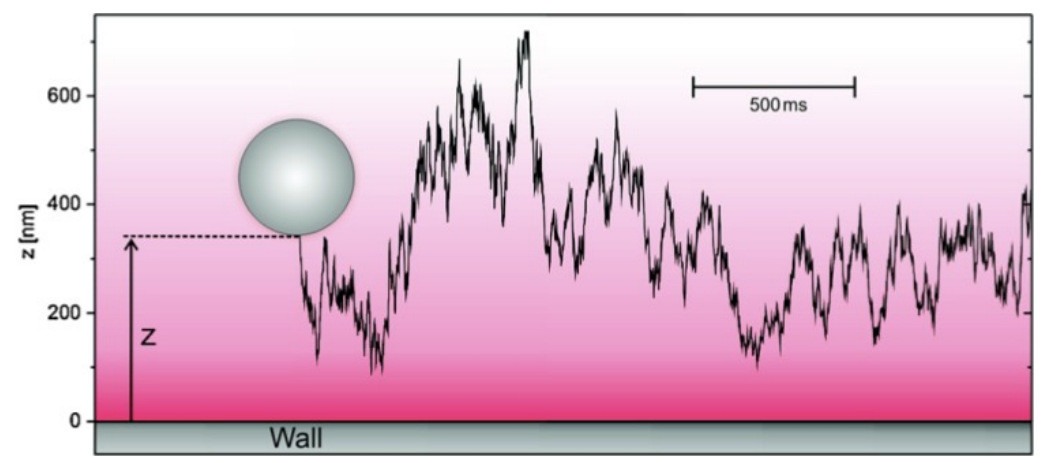

Figure 4. Brownian particle diffusing near a wall. A Brownian particle (drawn not to scale) diffuses near a wall. Its trajectory perpendicular to the wall is measured with TIRM using an evanescent electromagnetic field (red). 
Amongst various techniques available to probe the mechanical properties of microsystems, the strength of TIRM lies in its sensitivity to very weak interactions. Atomic Force Microscopy (AFM) requires a macroscopic cantilever as a probe and is typically limited to forces down to several piconewton $\left(10^{-12} \mathrm{~N}\right)$; the sensitivity of Photonic Force Microscopy (PFM) can even reach a few femtonewtons $\left(10^{-15} \mathrm{~N}\right)$, but this method is usually applied to bulk measurements far from any surface. TIRM, instead, can measure forces with femtonewton resolution acting on a particle near a surface. Over the last years, TIRM has been successfully applied to study electrostatic, van der Waals, depletion, magnetic, critical Casimir forces, and spurious forces ${ }^{26-36}$.

\section{FORCE MEASUREMENT IN DIFFUSION GRADIENTS}

Apart from the technological challenge intrinsic to dealing with forces in the order of femtonewton, it is important to realize that the general concepts we apply in our everyday life cannot be simply scaled down to microscopic objects, mainly due to the presence of thermal noise affecting the motion of small objects (Brownian motion ${ }^{1}$ ).

We demonstrated how the ineluctable presence of thermal noise alters the measurement of forces acting on microscopic and nanoscopic objects ${ }^{35-36}$. Our results demonstrated that the force-measurement process is prone to artifacts if the noise is not correctly taken into account. Indeed, the presence of a spatially varying Brownian noise leads to the presence of spurious forces, i.e. forces that exist only due to and in the presence of thermal noise. If overlooked, this leads to erroneous forces, which may severely affect the physical interpretations of experimental data. We quantified this effect exemplarily for a Brownian particle near a wall subjected to gravitational and electrostatic forces. These results are intimately connected to the long-standing issue of the interpretation of multiplicative noise in stochastic differential equations, i.e. the Ito-Stratonovic dilemma ${ }^{37-42}$.

The forces acting on a microscopic object immersed in a fluid medium can be assessed either by studying the underlying potential or by studying their effect on the object's trajectory ${ }^{36}$. The first approach - to which we shall refer as equilibrium distribution method - requires sampling of the equilibrium distribution. Accordingly, it can be only applied under conditions where the investigated system is at or close to thermodynamic equilibrium with a heat bath. The second method - to which we shall refer as non-equilibrium method or drift method - does not require the object to be at or even close to thermal equilibrium. Therefore, it can be applied also to systems which are intrinsically out-of-equilibrium, e.g., molecular machines, transport through pores, DNA stretching. The latter method, however, requires detecting the object trajectory with high sampling rates, which can be technologically more challenging, in particular when combined with a high spatial resolution. 


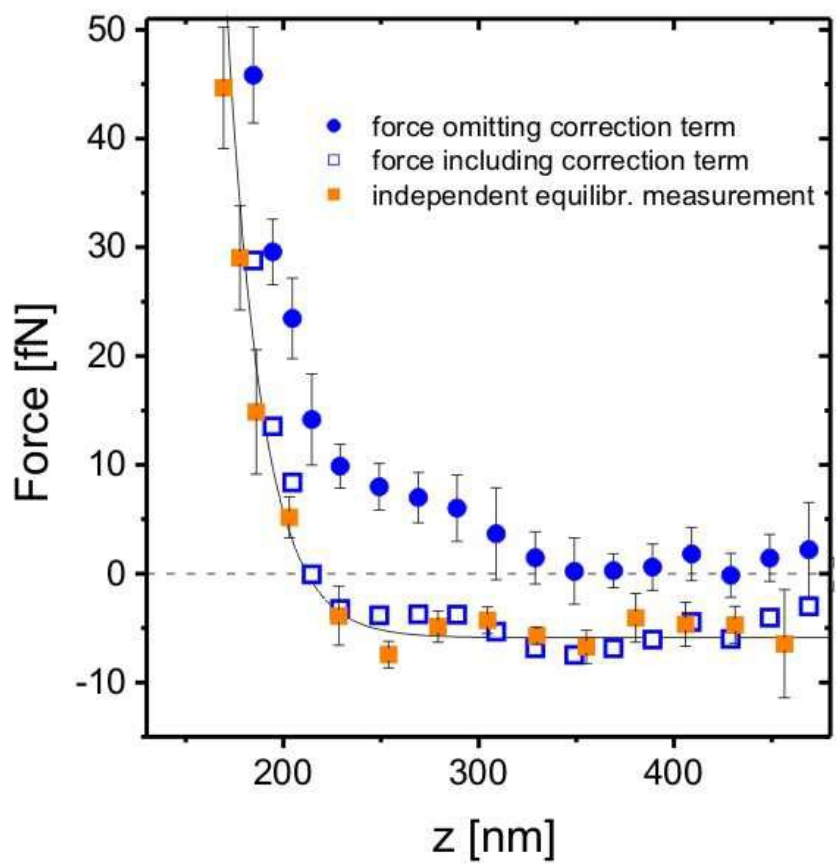

Figure 5. Equilibrium vs. nonequilibrium force measurement. Comparison of forces obtained from local drift velocity measurements of a Brownian particle (non-equilibrium measurements, bullets) and measured from the equilibrium particle height distribution (equilibrium measurements, solid squares). The difference is due to a spurious force arising from the diffusion gradient near the wall. Adapted from Ref. 35.

We have prepared a Mathematica demonstration focusing on a paradigmatic case of a Brownian particle in front of a wall $^{43}$. The user can explore the dependence of the spurious force term and the associated spurious drift on the particle radius and distance from the wall in water as medium. The correction terms become more relevant for smaller distances and radii. While the spurious drift reaches a constant value for $\mathrm{z}=0$, the spurious force diverges. For high values the spurious force is indicated by a green arrow. Note that in contrast to the spurious drift, the spurious force only depends on the particle radius and not on the viscosity of the medium.

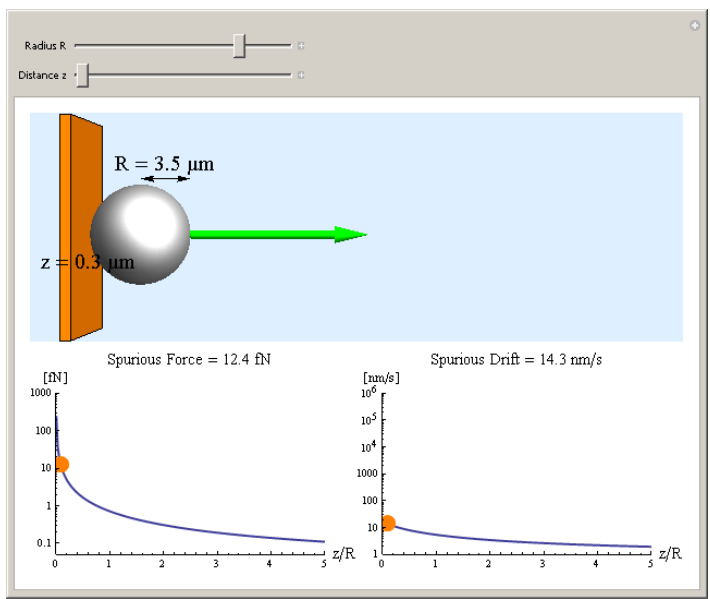

Figure 6. Spurious force and drift as a function of the wall-particle distance (Preview). The spurious force and drift for a 3.5 micron radius particle at 0.3 micron from a wall. The animation shows that both spurious drift and spurious force increase when the particle gets closer to the wall. See Ref. 43 to interact with the animation. 


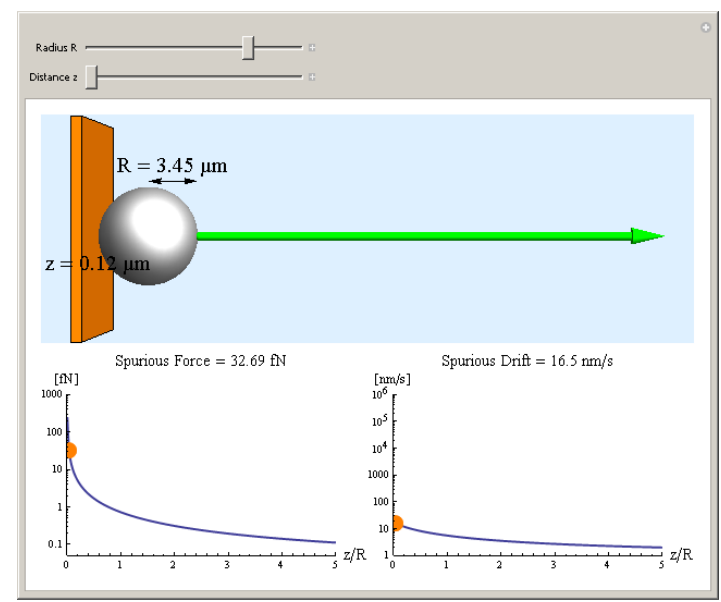

Figure 7. Spurious force and drift as a function of the particle radius (Preview). The spurious force and drift for a 3.45 micron radius particle at 0.12 micron from a wall. The animation demonstrates that at a given particle-wall separation the spurious force increases for larger particle, even though the spurious drift decreases. See Ref. 43 to interact with the animation.

\section{APPLICATIONS}

\subsection{Biophysics}

Biomolecules exert forces ${ }^{44-47}$ : the microtubule-based kinesin motors and actin-based myosin motors generate forces associated with intracellular trafficking, cell division, and muscle contraction; RNA polymerase during transcription exert a maximum force of about $14 \mathrm{pN}$. Also torques can be found in the microworld: flagellar motors - a complex of about 100 protein molecules - can rotate at more than 100 revolutions per second; and a single molecule of F-ATP synthase acts as a rotary motor, the smallest known yet. Knowledge of the mechanical properties of DNA is required to understand the structural dynamics of cellular processes such as replication and transcription. Measurements of force and extension on single molecules of DNA have allowed direct determination of its mechanical properties, provided rigorous tests of polymer elasticity theories, revealed unforeseen structural transitions induced by mechanical stresses, and established an experimental and conceptual framework for mechanical assays of enzymes that act on DNA. Forces and torques also appear in the presence of microfluidic flows, such as in lab-on-a-chip applications.

\subsection{Plasmonics}

Optical forces have become a powerful tool in physical, chemical, and especially biological experiments where an accurate and non-invasive manipulation of small objects from the nanometer to the micrometer scale is required. Within the current general trend for miniaturization, the implementation of the so-called "optical tweezers" (OT) at a surface remains today a key aspiration which would open a huge potential for future reconfigurable lab-on-a-chip devices entirely operated with light. The transition from 3D to $2 \mathrm{D}$ optical manipulation is made possible by exploiting evanescent fields bound at interfaces. In particular, surface plasmons (SP) bound at metal surfaces are expected to lead to key breakthroughs. SP are surface modes associated to a resonant oscillation of the surface charges of the metal with the incident electromagnetic field. They result in a multifold increase of the surface field strength and potentially allow for scaling trapping volumes down to the nanometer scale.

We provided the first experimental demonstration of the plasmon optical forces ${ }^{48}$. The enhanced force field experienced by a single micrometer bead exposed to the SP at a flat metal/dielectric interface has been for the first time measured using a Photonic Force Microscope. The total force magnitude was found to be 40 times stronger compared to otherwise, without feeding the SP. The simplicity and flexibility of SP-based optical manipulation pave the way toward a new 
generation of analytical devices where the analyte would be manipulated, arranged and analyzed at the chip surface for low cost and parallel bio-analysis ${ }^{49-51}$.

\subsection{Statistical Physics}

The study of noisy phenomena is one of the most fascinating branches of statistical physics. Noisy systems are ubiquitous in natural and engineered phenomena. The presence of noise becomes particularly evident when we move down into molecular-scale phenomena: the thermal noise, responsible for the Brownian diffusion of particles, is omnipresent. However, noise is also intrinsic to many macroscopic systems: stock markets, population dynamics, ecosystems and traffic flows, all present some degree of noise.

Colloidal particles, i.e. microscopic particles suspended in a fluid, are often referred to as model systems to address novel concepts in the context of statistical physics in a convenient way ${ }^{52}$. This is particularly true for the study of noisy phenomena, because colloidal particles are constantly moving due to the presence of thermal fluctuations. Such fluctuations - responsible for the diffusion of the Brownian particles - introduce a well-defined noisy background.

The presence of some deterministic forces acting on the particles can affect the random walk of the particles producing a drift. It is possible to make use of optical forces to introduce deterministic perturbations acting on the particle in a very controllable way.

Optically trapped particles can, therefore, be a very powerful tool to study statistical physics phenomena, relying both on the presence of a natural noisy background and on a finely controllable deterministic force field. Optically trapped particles have been used to study experimentally more non-trivial, sometimes constructive, aspects of the presence of noise, e.g., stochastic resonance, stochastic resonant damping, stochastic activation, nanoscopic thermal motors, and Brownian ratchets ${ }^{53-57}$.

\subsection{Active Microswimmers}

In recent years, active Brownian motion has attracted a lot of interest ${ }^{58}$. This is a way to generate forces that act on the nanoscale. Differently from simple Brownian motion, which is dominated by random fluctuations, active Brownian particles, e.g. bacteria, feature an interplay between random fluctuations and active swimming. Various model systems have been proposed for its study, e.g. Janus particles in a water-hydrogen peroxide. We proposed a novel model systems where the swimming behavior of the particles can be tuned by the intensity of illumination ${ }^{59-60}$. We showed how this leads to the possibility of studying the behavior of active Brownian particles under new conditions, e.g. swimming behavior depended on the particle position, and in new environments, e.g. non-homogenous and porous media. We also show how this may have applications for the study of the behavior of bacteria.

\section{REFERENCES}

[1] Nelson, E., [Dynamical Theories of Brownian Motion], Princeton University Press, Princeton (1967).

[2] Happel, J. and Brenner, H., [Low Reynolds Number Hydrodynamics], Springer, New York (1983).

[3] Purcell, E. M., "Life at low Reynolds numbers," Am. J. Phys. 45, 3-11 (1977).

[4] Binnig, G., Rohrer, H., Gerber, C. and Weibel, E., "Surface studies by scanning tunneling microscope," Phys. Rev. Lett. 49, 57-60 (1982).

[5] Binnig, G., Quate, C. F. and Gerber, C., “Atomic force microscope,” Phys. Rev. Lett. 56, 930-933 (1986).

[6] Ashkin, A., Dziedzic, J. M., Bjorkholm, J. E. and Chu, S., "Observation of a single-beam gradient force optical trap for dielectric particles," Opt. Lett. 11, 288-290 (1986).

[7] Ghislain, L. P. and Webb, W. W., "Scanning-force microscope based on an optical trap," Opt. Lett. 18, 16781680 (1993).

[8] Weisenhorn, A. L., Hansma, P. K., Albrecht, T. R., and Quate, C. F., "Forces in atomic force microscopy in air and water," Appl. Phys. Lett. 54, 2651-2653 (1989).

[9] Berg-Sørensen K. and Flyvbjerg, H., "Power spectrum analysis for optical tweezers," Rev. Sci. Instrum. 75, 594-612 (2004). 
[10] Volpe, G., Kozyreff, G. and Petrov, D., "Back-scattering position detection for photonic force microscopy," J. Appl. Phys. 102, 084701 (2007).

[11] Volpe, G. and Petrov, D., "Torque detection using Brownian fluctuations," Phys. Rev. Lett. 97, 210603 (2006).

[12] Volpe, G., Volpe, G. and Petrov, D., "Brownian motion in a nonhomogeneous force field and photonic forcemicroscope," Phys. Rev. E 76, 061118 (2007).

[13] La Porta, A. and Wang, M. D., "Optical torque wrench: Angular trapping, rotation, and torque detection of quartz microparticles,” Phys. Rev. Lett. 92, 190801 (2004).

[14] Rohrbach, A., and Stelzer, E. H. K., "Three-dimensional position detection of optically trapped dielectric particles," J. Appl. Phys. 91, 5474-5488 (2002).

[15] Rohrbach, A., "Switching and measuring a force of 25 femtonewtons with an optical trap," Opt. Express 13, 9695-9701 (2005).

[16] Volpe, G., Volpe, G. and Petrov, D., "Singular point characterization in microscopic flows," Phys. Rev. E 77, 037301 (2008).

[17] Borghese, F., Denti, P., Saija, R., Iati, M.A., Maragò, O.M., "Radiation torque and force on optically trapped linear nanostructures," Phys. Rev. Lett. 100, 163903 (2008).

[18] Jones P. H.; Palmisano F.; Bonaccorso F.; Gucciardi P. G.; Calogero G.; Ferrari A. C.; Maragò O. M., "Rotation Detection in Light-Driven Nanorotors," ACS Nano 3, 3077-3084 (2009).

[19] Pesce, G., Volpe, G., De Luca, A. C., Rusciano, G. and Volpe, G., "Quantitative assessment of nonconservative radiation forces in an optical trap," EPL (Europhys. Lett.) 86, 38002 (2009).

[20] Pesce, G., Volpe, G., Imparato, A., Rusciano, G., and Sasso, A., "Influence of rotational force fields on the determination of the work done on a driven Brownian particle," J. Opt. 13, 044006 (2011).

[21] Volpe, G., Wehr, J., Petrov, D. and Rubi, J. M., "Thermal Noise Suppression: How Much Does It Cost?" J. Phys. A: Math. Theor. 42, 095005 (2009).

[22] Huang, R., Chavez, I., Taute, K. M., Lukic, B., Jeney, S., Raizen, M. G., and Florin, E.-L., "Direct observation of the full transition from ballistic to diffusive Brownian motion in a liquid," Nat. Phys. 7, 576 (2011).

[23] Walz, J., "Measuring particle interactions with total internal reflection microscopy," Curr. Opin. Colloid. Interface Sci. 2, 600-606 (1997).

[24] Prieve, D. C., "Measurement of colloidal forces with TIRM," Adv. Colloid. Interface Sci. 82, 93-125 (1999).

[25] Volpe, G., Brettschneider, T., Helden, L. and Bechinger, C., "Novel perspectives for the application of total internal reflection microscopy," Opt. Express 17, 23975-23985 (2009).

[26] Bike, S. G. and D. C. Prieve, D. C., "Measurements of double-layer repulsion for slightly overlapping counterion clouds," Int. J. Multiphase Flow 16, 727-740 (1990).

[27] von Grünberg, H. H., Helden, L., Leiderer, P. and Bechinger, C., "Measurement of surface charge densities on Brownian particles using total internal reflection microscopy," J. Chem. Phys. 114, 10094-10104 (2001).

[28] Bevan, M. A. and Prieve, D. C., "Direct measurement of retarded van der Waals attraction,” Langmuir 15, 79257936 (1999).

[29] Piech, M., Weronski, P., Wu, X. and Walz, J. Y., "Prediction and measurement of the interparticle depletion interaction next to a flat wall," J. Colloid Interface Sci. 247, 327-341 (2002).

[30] Bevan, M. A. and Scales, P. J., "Solvent quality dependent interactions and phase behavior of polystyrene particles with physisorbed PEO-PPO-PEO," Langmuir 18, 1474-1484 (2002).

[31] Helden, L., Roth, R., Koenderink, G. H., Leiderer, P. and Bechinger, C., "Direct measurement of entropic forces induced by rigid rods," Phys. Rev. Lett. 90, 048301 (2003).

[32] Kleshchanok, D., Tuinier, R. and Lang, P. R., "Depletion interaction mediated by a polydisperse polymer studied with total internal reflection microscopy," Langmuir 22, 9121-9128 (2006).

[33] Blickle, V., Babic, D. and Bechinger, C., "Evanescent light scattering with magnetic colloids," Appl. Phys. Lett. 87, 101102 (2005).

[34] Hertlein, C., Helden, L., Gambassi, A., Dietrich, S. and Bechinger, C., "Direct measurement of critical Casimir forces," Nature 451, 172-175 (2008).

[35] Volpe, G., Helden, L., Brettschneider, T., Wehr, J. and Bechinger, C., "Influence of Noise on Force Measurements," Phys. Rev. Lett. 104, 170602 (2010).

[36] Brettschneider, T., Volpe, G., Helden, L., Wehr, J., Bechinger, C., "Force measurement in the presence of Brownian noise: Equilibrium distribution method vs. drift method," Phys. Rev. E 83, 041113 (2011).

[37] Van Kampen, N. G., [Stochastic Processes in Physics and Chemistry], North-Holland, Amsterdam (1981).

[38] Karatzas, I., and Shreve, S., [Brownian Motion and Stochastic Calculus], Springer, New York (1998). 
[39] Øksendal, B., [Stochastic Differential Equations], Springer, Berlin (1998).

[40] Ermark, D.L., and McCammon, J.A., "Brownian dynamics with hydrodynamic interactions," J. Chem. Phys. 69, 1352 (1978).

[41] Lau, A.W.C., and Lubensky, T.C., "State-dependent diffusion: Thermodynamic consistency and its path integral formulation," Phys. Rev. E 76, 011123 (2007).

[42] Hottovy, S., Volpe, G., and Wehr, J., "Spurious drift in stochastic differential equations with arbitrary friction and diffusion in the Smoluchowski-Kramers limit," J. Stat. Phys. 146, 762-773 (2012).

[43] Brettschneider, T., and Volpe, G., "Spurious Forces on a Brownian Particle in a Diffusion Gradient," $<$ http://demonstrations.wolfram.com/SpuriousForcesOnABrownianParticleInADiffusionGradient/>.

[44] Berry, R. M. and Berg, H. C., "Absence of a barrier to backwards rotation of the bacterial flagellar motor demonstrated with optical tweezers," Phys. Rev. Lett. 94, 14433 (1997).

[45] Simpson, N. B., Dholakia, K., Allen, L. and Padjett, M. J., "Mechanical equivalence of spin and orbital angular momentum of light: an optical spanner," Opt. Lett. 22, 52 (1997).

[46] Ryu, W. S., Berry, R. M. and Berg, H. C., "Torque-generating units of the flagellar motor of escherichia coli have a high duty," Nature 403, 444 (2000).

[47] Bryant, Z., Stone, M. D., Gore, J., Smith, S. B., Cozzarelli, N. R. and Bustamante, C., "Structural transitions and elasticity from torque measurements on DNA," Nature 424, 338 (2003).

[48] Volpe, G., Quidant, R., Badenes, G. and Petrov, D., "Surface plasmon radiation forces," Phys. Rev. Lett. 96, 238101 (2006).

[49] Righini, M., Zelenina, A. S., Girard, C. and Quidant, R., "Parallel and selective trapping in a patterned plasmonic landscape," Nat. Phys. 3, 477-480 (2007).

[50] Righini, M., Volpe, G., Girard, C., Petrov, D. and Quidant, R., "Surface plasmon optical tweezers: tunable optical manipulation in the femto-newton range," Phys. Rev. Lett. 100, 186804 (2008).

[51] Volpe, G., Volpe, G., and Quidant, R., "Fractal plasmonics: subdiffraction focusing and broadband spectral response by a Sierpisky nanocarpet," Opt. Express 19, 3612-3618 (2011).

[52]Babic, D., Schmitt, C. and Bechinger, C., "Colloids as model systems for problems in statistical physics," Chaos 15, 026114 (2005).

[53] Gammaitoni, L., Hanggi, P., Jung, P. and Marchesoni, F., “Stochastic resonance," Rev. Mod. Phys. 70, 223-287 (1998).

[54] Doering, C. R. and Gadoua, J. C., "Resonant activation over a fluctuating barrier," Phys. Rev. Lett. 69, 23182321, (1992).

[55] Astumian, R. D., "Thermodynamics and kinetics of a brownian motor," Science 276, 917-922 (1997).

[56] Volpe, G., Perrone, S., Rubi, J. M. and Petrov, D., "Stochastic Resonant Damping in a Noisy Monostable System: Theory and Experiment," Phys. Rev. E 77, 051107 (2008).

[57] Volpe, G., Wehr, J., Petrov, D. and Rubi, J. M., "Thermal Noise Suppression: How Much Does It Cost?” J. Phys. A: Math. Theor. 42, 095005 (2009).

[58] Ebbens, S. J., and Howse, J. R., "In pursuit of propulsion at the nanoscale," Soft Matter 6, 726-738 (2010).

[59] Volpe, G., Buttinoni, I., Vogt, D., Kümmerer, H. J., and Bechinger, C., "Microswimmers in patterned environments," Soft Matter 7, 8810-8815 (2011).

[60] Buttinoni, I., Volpe, G., Kümmel, F., Volpe, G., and Bechinger, C., "Active Brownian motion tunable by light," J. Phys.: Condens. Matter 24, 284129 (2012). 